Erschienen in: Stickel, Gerhard (Hrsg.): Deutsche Gegenwartssprache. Tendenzen und Perspektiven. - Berlin, New York: de Gruyter, 1990. S. 184-191. (Institut für deutsche Sprache. Jahrbuch 1989)

\title{
RENATE HERRMANN-WINTER
}

\section{Standardsprache und Mundarten in der Deutschen Demokratischen Republik}

Gestatten Sie mir zunächst die Vorbemerkung, daß Standard, Dialekt und die sogenannten Umgangssprachen, von deren Verwendung in der DDR und vor allem im Norden unseres Landes die Rede sein soll, hier als linguistische Begriffe nicht theoretisch reflektiert werden können, ebensowenig wie die damit verbundenen Sprachgliederungsmodelle.

\section{Verwendung der Standardsprache}

In der Zunahme der Standardverwendung und dem Rückgang der Dialekte während der zurückliegenden vier Jahrzehnte setzte sich einerseits eine durch Industrie, Verkehr und Wissenschaft seit dem 19. Jahrhundert vorangetriebene Entwicklung fort. Andererseits hatten die gesellschaftlichen Umwälzungen in der DDR auch veränderte Bedingungen für den Gebrauch der Dialekte, Umgangssprachen und des Standards geschaffen. Standardgebrauch war alleiniges Anliegen der Sprachpflege, bis dann in den 60iger Jahren die situationsangemessene Verwendung aller Varietäten gefordert wurde. Aber auch die situative Variationsfähigkeit setzt möglichst hohe Standardkenntnisse voraus. Mit diesem Wandel korrespondierte eine Erweiterung der Normen vor allem für den gesprochenen Standard: Unterschiede zwischen reproduktivem und produktivem Sprechen werden berücksichtigt ebenso wie situative Gesprächsformen, aber die regionale Neutralität der Standardlautung bleibt als Postulat bestehen.

Es existieren heute noch weithin beträchtliche Unterschiede in der Standardbeherrschung, abhängig von Bildungsniveau und Alter, auch von der regional-sprachlichen Herkunft und den jeweiligen Bedürfnissen und Anforderungen in der Kommunikation. Dennoch hat sich insgesamt gesehen der Einfluß des Standards auf die sogenannte sprachliche Normallage und damit auch auf die inoffizielle Alltagskommunikation verstärkt. Als ein Indiz dafür sei angeführt, daß der Anteil standardsprachlicher Substantive in weitgehend alltagssprachlichen Texten der Mitte 70iger Jahre aus dem Kreis Greifswald $95 \%$ betrug. Der Anteil DDR-typischer Neologismen war mit $10 \%$ an der Gesamtmenge von 10.000 verwendeten Substantiven doppelt so hoch wie der des regionalen Nichtstandards.

Auch in der Familie wird zunehmend der Gebrauch einer standardnahen Sprachform angestrebt, und es geschieht vielfach mit Rücksicht auf die Kinder. In allen Sprachlandschaften der DDR wird generell - spätestens 
seit Mitte der 60iger Jahre - Dialekt nicht mehr als Erstsprache gelernt. Beim mündlichen Standardgebrauch werden auch in offentlicher Rede oder beim Gespräch in offiziellen Situationen die Standardnormen auf der phonetischen Ebene zumeist nicht erreicht und vielfach auch nicht angestrebt. Geschriebener Standard - durch zunehmende Verschriftlichung der sprachlichen Kommunikation gefördert - zeichnet sich demgegenüber durch ein höheres Korrektheitsstreben, vor allem in bezug auf die orthographischen Normen aus. Sie bestimmen auch den schriftlichen Nichtstandard, z.B. in der umgangssprachlichen privaten Korrespondenz und beeinflussen die Schreibweisen der Dialekte. Andererseits haben das tolerantere Normenverständnis und das neue öffentliche Prestige der Dialekte und Umgangssprachen Auswirkungen auf traditionelle Domänen des mündlichen und schriftlichen Standards. Verstärkt dringen z.B. in öffentliche Rede oder in die Presse nichtstandardsprachliche Elemente ein, auch als Mittel stilistischer Wirkung und Träger pragmatischer Informationen. So wird trotz der Ausbreitung des Standards eine sprachliche Heterogenität fortbestehen, und Regionalismen werden möglicherweise noch zunehmen.

\section{Verwendung von Mundarten und Umgangssprachen}

Zur Verwendung von unterschiedlichen regionalen Sprachformen sind relativ gesicherte Aussagen über die historisch gewachsene dialektgeographische Verteilung möglich. Hier sei nur darauf hingewiesen, daß sich das Niederdeutsche mit den mecklenburg-vorpommerschen, brandenburgischen und ostfälischen Mundarten von der Küste bis etwa zu der Linie Magdeburg-Wittenberg-Beeskow (ca. $60 \mathrm{~km}$ südöstlich von Berlin gelegen) erstreckt und somit in der DDR den größten Raum einnimmt. Dagegen gibt es oberdeutsche Mundarten nur in zwei Randzonen, im thüringischen Bezirk Suhl sowie im Vogtland und im Westerzgebirge. Weniger klar ist schon die Abgrenzung und Verteilung der sogenannten regionalen Umgangssprachen. Erhebungen dazu, die das gesamte Territorium der DDR umfassen, sollen in diesem Jahr abgeschlossen werden, doch die computative Auswertung steht noch bevor.

Gemeinhin werden heute drei bzw. fünf großräumige Umgangssprachen mit ihren jeweiligen Abstufungen unterschieden: die mecklenburgische, die berlin-brandenburgische und die obersächsisch-thüringische, bzw. zusätzlich eine südwest- und westthüringische. Sie überdachen eine größere Zahl mittlerer und kleinräumiger Umgangssprache. Die zwischen ihnen bestehenden lautlichen und intonatorischen Unterschiede - verursacht durch dialektale Substrate oder ausstrahlende Stadtdialekte sind heute noch gewichtiger als die allen Umgangssprachen gemeinsamen 
Merkmale. Die Tendenz zur Ausbildung einer einheitlichen Umgangssprache in der DDR, die man noch vor 15 Jahren zu erkennen glaubte, hat sich nicht bestätigt.

Aus diachronischer Sicht können auf dem Gebiet der DDR, für das die Dialektgeographie einstmals 42 kleinräumige Mundartlandschaften ermittelt hat, heute vielleicht drei Entwicklungstypen in der regionalen Sprachverwendung unterschieden werden:

1. Gebiete, in denen die entscheidende Ablösung der Mundarten in der Alltagskommunikation bereits vor 1945 erfolgte.

2. Gebiete, innerhalb derer in Dörfern und Kleinstädten diese Wende vor allem in den 50iger und 60iger Jahren erfolgte, und

3. Gebiete, in denen auf dem Lande ein nennenswerter Rückgang der Mundarten erst in unserem Jahrzehnt beginnt.

Beispiel für den zuletzt genannten Typ ist der Bezirk Suhl. Südlich des Thüringer Waldes verwenden heute noch mehr als $70 \%$ der Bewohner ländlicher Gemeinden das fränkische Hennebergische (Itzgründische) in öffentlichen Situationen: bei der Arbeit, beim Einkauf, in der Gaststätte, bei dörflichen Festen oder auch im Gemeindebüro. In den größeren Städten (Suhl und Meiningen) dagegen überwiegt die mainfränkisch geprägte Umgangssprache, und der gelegentliche Mundartgebrauch beschränkt sich auf die ältere Generation.

Beispiel für den zweiten Typ sind: die mitteldeutschen Randgebiete Thüringens, wie das Eichsfeld und der Südharz, oder Dörfer in der Magdeburger Börde und in der Altmark, oder die ländlichen Gebiete einschließlich der Kleinstädte in den Bezirken Schwerin, Rostock und Neubrandenburg. Hier ist die Mundart trotz eines entscheidenden Rückganges im Vergleich zur Situation vor 1945 auch heute noch für eine nennenswerte $Z$ ahl von Sprechern ein wichtiges Mittel in der Kommunikation.

Beispiel für den 3. Typ wäre der südliche niederdeutsche, also brandenburgische Raum, wo sich schon zu Beginn unseres Jahrhunderts durch den Einfluß Berlins und Obersachsens Umgangssprachen mit niederdeutschen und mitteldeutschen Interferenzmerkalen durchzusetzen begannen. Die besondere Ausstrahlungskraft des Berlinischen hält bis heute an. Vor allem aber gehören dazu Teile des obersächsischen Sprachraumes etwa von Halle bis Görlitz einschließlich Zentralthüringens. Hier haben die sich von den Industrielandschaften ausbreitenden Umgangssprachen die Mundarten bereits seit Beginn des 20. Jahrhunderts verdrängt, zersetzt und aufgelöst. Das hatte zur Folge, daß regional zwischen Grunddialekt und Standard ein Kontinuum schwer von einander abgrenzbarer 
Sprachformen, aber keine Diglossie-Situation entstand. Die obersächsischen Umgangssprachen wurden normprägend für die mündliche Kommunikation in diesen Gebieten. Im Sprecherbewußtsein haben sie vielfach den Status von Mundarten.

\section{Verwendung regionaler Sprachformen im Norden (Domänen, soziale Geltung, Entwicklung)}

Ich komme zurück auf den mecklenburgisch-vorpommerschen Sprachraum. Hier dominiert heute in allen Bereichen der mündlichen Kommunikation Hochdeutsch in norddeutscher Prägung. Es weist in Abhängigkeit von Bildungsniveau und Alter mehr oder weniger starke regionale Abstufungen und Abweichungen von der Standardsprache auf (in standardnaher Ausprägung weniger in der Lautung, mehr in der Lexik und Grammatik). Diese sogenannte mecklenburgische oder norddeutsche Umgangssprache wird gemeinhin im Sprecherbewußtsein nicht vom Standard unterschieden.

Die zunehmende Verbreitung des Hochdeutschen seit 1945 wurde nicht nur durch den vergleichsweise hohen Anteil der Umsiedler (in manchen Dörfern bis zu $60 \%$ ) gefördert. Allerdings lernten damals viele jüngere Umsiedler und deren Kinder auf dem Lande noch die Mundart ihrer neuen Heimat klangecht sprechen. Hinzu kam der Zustrom von Arbeitskräften aus dem berlinisch-brandenburgischen und vor allem den mitteldeutschen Sprachräumen, der sogenannten "Nordlandfahrer” beim Aufbau der neuen Industriezentren und der Umgestaltung der Landwirtschaft.

Der Rückgang des Niederdeutschen wurde nicht zuletzt durch die offiziell negative Bewertung des Dialektes in den 50iger und 60iger Jahren vorangetrieben. Nur-Mundartsprecher gibt es heute nicht mehr. Gegenüber der abnehmenden Zahl aktiver Mundartsprecher ist seit Mitte der 70iger Jahre eine Zunahme von passiven Mundartkenntnissen zu beobachten. Verstehen heute schätzungsweise wohl wenigstens $60 \%$ der Bevölkerung zumindest ausreichend Niederdeutsch, schwankt der Anteil der aktiven Sprecher von vielleicht $10 \%$ in den größeren Städten, etwa $30 \%$ in ländlichen Gebieten und Kleinstädten bis zu etwa $80 \%$ in wenigen Reliktgebieten.

Die Neubewertung der Mundart und zahlreiche Aktivitäten im kulturellen Bereich haben - durch Medien verbreitet - zur Erweiterung der passiven Niederdeutschkenntnisse auch südlich der Nordbezirke beigetragen. Unter den Mundartsprechern dominieren heute noch die Bauern, Fischer, Arbeiter und Handwerker. Aber auch Angehörige aus allen ande- 
ren sozialen Schichten, nicht zuletzt der Intelligenz, gehören dazu, und sie engagieren sich in besonderem Maße für die Förderung des Niederdeutschen. So wird heute im Norden - anders als etwa in Zentralthüringen Mundartgebrauch nicht mehr pejorativ und nicht als soziales Phänomen bewertet.

Entscheidender als die soziale Markierung der Sprecher ist das Alter. Die meisten aktiven Mundartsprecher sind heute älter als 50, darunter mehr Männer als Frauen. Die Zahl der mundartkundigen Schüler ging Jahrzehnte kontinuierlich zurück, jetzt steigt sie durch schulische Bemühungen wieder leicht an. Gelernt wird heute auch Plattdeutsch nicht mehr als erste Sprache und immer seltener in der eigenen Elternfamilie, vielmehr zunächst passiv als $Z$ weitsprache in der Grobfamilie.

Ohne die negativen Konsequenzen des Übergangs zum Zweiterwerb überbetonen zu wollen, sei auf folgendes hingewiesen: Der Ersterwerb in der Familie führte dazu, daß für bestimmte Situationstypen, z.B. das Gespräch mit Geschwistern, die Mundartverwendung fast obligatorisch wurde, und der Ersterwerb bewirkte zudem eine umfassendere dialektale Kompetenz. Wenn aktives Niederdeutsch erst in Arbeitskollektiven, in Freundes- oder Freizeitgruppen erlernt wird, so kann der Erfolg - weil motiviert - ebenso groß sein wie beim Ersterwerb, aber in der Regel wird ein weitaus geringeres Dialektniveau erreicht.

Die stärksten Motivationen für den sekundären Dialekterwerb gehen heute im Norden der DDR von Arbeitsbereichen wie Land-, Forst- und Bauwirtschaft, den Werften, von industriellen und handwerklichen Kleinbetrieben aus, wo Niederdeutsch noch die größte Geltung hat. Ebenso behauptet sich Niederdeutsch weiterhin in anderen Alltagssituationen, in der Groffamilie und im Freundes- und Bekanntenkreis, weil es in besonderer Weise menschliche Kontakte, emotionale Beziehungen und Gruppenidentität zu fördern vermag. Partnerbeziehungen lösen Mundartverwendung auch in Situationen aus, in denen allgemein Hochdeutsch üblich ist. Der Stadt-Land-Unterschied ist für den Mundartgebrauch in Mecklenburg-Vorpommern weitaus weniger wichtig als im mitteldeutschen Sprachraum.

Die Domänenerweiterung des Niederdeutschen im kulturellen Leben führte zu einem Zuwachs an schriftlicher Mundartverwendung in der Presse, in der Werbung, in heimatkundlichen Broschüren und nicht zuletzt in der beachtlich angestiegenen Buchproduktion von niederdeutscher Gegenwartsprosa bis zur Bibelübersetzung. Anders als beim Sprechen bilden sich im schriftlich ausgebauten, literarisierten Niederdeutsch ansatzweise textsortenspezifische Stile aus. 
Dieses Auseinanderdriften von mündlichem und schriftlichem Dialektgebrauch wird im Sprecherbewußtsein reflektiert und z.B. bei der Presse als „Zeitungsplatt" nicht immer positiv bewertet. Solche Urteile wurzeln nicht in einem niederdeutschen Purismus, denn hochdeutschniederdeutsche Interferenz und Alternanz sind im Alltagsgespräch üblich und selbstverständlich. Niederdeutsch ist für die Mehrzahl der Sprecher eine Sprache und keine Mundart. Es hat in ihrem Bewußtsein nicht nur eine mit Hochdeutsch gleichrangige Position, vielmehr eine besondere, die Gefühl, menschliche Wärme und Heimat assoziiert. Dieses dialektale Selbstbewußtsein wird aus einem - zumindest in elementarer Form weitverbreiteten - Wissen über die bedeutende sprachlich-kulturelle Vergangenheit des Niederdeutschen gespeist. Auch daraus erklärt sich seine Sonderstellung unter den Dialekten in der DDR.

Es könnten für das Niederdeutsche noch weitere sogenannte dialektstabilisierende Faktoren genannt werden. Dennoch steht es nicht außerhalb der allgemeinen Trends von Standard- und Dialekt-Verwendung in der DDR, das heißt: Zunahme des Standards - grundsätzlich in allen Verwendungsbereichen - mit toleranteren Normierungen und dem Anwachsen von Nichtstandard-Interferenzen; das heißt: Rückgang der Dialekte durch sinkende Gebrauchshäufigkeit und Dialektalitätsabbau; das heißt aber auch steigender Einfluß von Dialekten und Umgangssprachen in traditionellen Standarddomänen. Diese Grundtendenzen werden auch künftig regional geprägt und modifiziert.

\section{Literatur}

Aktuelle Probleme (1974): Aktuelle Probleme der sprachlichen Kommunikation. Soziolinguistische Studien zur sprachlichen Situation in der Deutschen Demokratischen Republik. Berlin.

Bergmann, Gunter (1974): Sprachschichtung und Sprachwandel - dargestellt an den Entwicklungen der Vokalphoneme im Gebiet um Karl-MarxStadt. In: Beiträge zur Soziolinguistik. Halle, S. 84-98.

Entwicklungstendenzen (1988): Entwicklungstendenzen in der deutschen $\mathrm{Ge}$ genwartssprache. Hrsg. v. K.-E. Sommerfeldt. Leipzig.

Gernentz, Hans Joachim (1980): Niederdeutsch - gestern und heute. Rostock.

Hartung, Wolfdietrich (1987): Sprachnormen - ihr sozialer Charakter und die linguistische Begrifflichkeit. In: ZPSK 40, S. 317-335.

Herrmann-Winter, Renate (1979): Studien zur gesprochenen Sprache im Norden der DDR. Berlin. 
Herrmann-Winter, Renate (1980): Wortgut aus niederdeutschen Pressetexten der DDR im Urteil von Mundartsprechern. In: Linguistische Studien des ZISW der AdW der DDR, R. A 75/I, S. 96-104.

Herrmann-Winter, Renate (1985): Urteile über Niederdeutsch in den Nordbezirken der DDR. In: ZPSK 38, S. 297-308.

Herrmann-Winter, Renate (1987): Zum Zusammenhang von Verwendungsweisen und Bewertungen des Niederdeutschen im Norden der DDR. In: ZPSK 40, S. 822-829.

Kommunikation (1981): Kommunikation und Sprachvariation. Autorenkollektiv u. Ltg. v. W. Hartung u. H. Schönfeld. Berlin.

Langner, Helmut (1984): Zum Einfluß der Umgangssprache auf die Literatursprache der Gegenwart. In: ZPSK 37, S. 191-199.

Nerius, Dieter (1987/88): Entwicklungstendenzen der deutschen Sprache in der DDR. In: Brücken. Germanistisches Jahrbuch DDR-ČSR, S. 270-291.

Schönfeld, Helmut (1974): Gesprochenes Deutsch in der Altmark. Berlin.

Schönfeld, Helmut (1986): Die berlinische Umgangssprache im 19. und 20. Jahrhundert. In: Berlinisch. Hrsg. v. Joachim Schildt und Hartmut Schmidt, Berlin, S. 214-289.

Schönfeld, Helmut (1987): Die Veränderungen in der Sprache und im sprachlichen Verhalten der Dorfbevölkerung. In: Das Leben der Werktätigen in der Magdeburger Börde, hrsg. v. H.-J. Rach, B. Weissel u. H. Plaul. Berlin.

Spangenberg, Karl (1970): Sprachwandel im thüringischen Eichsfeld. In: Wiss. Beitr. der F.S.-Univ. Jena, S. 202-224.

Spangenberg, Karl (1985): Zur Erforschung des hennebergischen Sprachraums. In: Wiss. Ztschr. F. S.-Univ. Jena Gesellschaftswiss. R. Jg. 34, H. 1 S. 21-35.

Spangenberg, Karl (1986): Untersuchungen zur Funktion, Geltung und Bewertung der Mundart in Thüringen. In: Beitr. zur Erforschung der dt. Sprache 6, S. 234-245.

Umgangssprachen (1986): Umgangssprachen und Dialekte in der DDR. Veröff. der F. S. - Univ. Jena. 
Wortschatz (1987): Wortschatz der deutschen Sprache in der DDR. Autorenkoll. u. Ltg. v. W. Fleischer. Leipzig. 